\title{
ESTUDIO DE PROPUESTAS INTERMEDIAS DE BALONCESTO EN CATEGORÍA INFANTIL
}

\section{STUDY ON INTERMEDIATE PROPOSALS FOR BASKETBALL IN THE CHILDREN'S CATEGORY}

\section{Garzón, Belén; Lapresa, Daniel; Anguera, María Teresa y Arana, Javier}

\author{
Doctora Belen Garzon Echevarría: blngarzon@gmail.com \\ Doctor Daniel Lapresa Ajamil: daniel.lapresa@unirioja.es \\ Doctora María Teresa Anguera Argilaga: mtanguera@gmail.com \\ Doctor Javier Arana Idiakez: katrapuna@gmail.com
}

Código UNESCO: 5802.99

Clasificación Consejo de Europa: 5

Este estudio ha sido financiado por el proyecto "Observación de la interacción en el deporte y actividad física: Avances técnicos y metodológicos en registros automatizados cualitativos-cuantitativos" (Secretaría de Estado de Investigación, Desarrollo e Innovación del Ministerio de Economía y Competitividad DEP2012-32124).

Recibido el 30 de marzo de 2014

Aceptado el 7 de abril de 2014

PALABRAS
CLAVE:
Baloncesto,
Adaptación,
Tiro libre,
$12-13$ años,
Patrones
temporales

\section{KEY WORDS:}

Basquetball,

Adaptation,

Free throw shot,

12-13 years

category,

T-patterns

\section{RESUMEN}

El presente trabajo pretende elevar una propuesta intermedia de baloncesto en la categoría infantil, que permita que el paso de la modalidad de minibasket al baloncesto adulto sea progresivo. Con tal objetivo se someten a estudio los efectos en el patrón técnico de lanzamiento de tiro libre de cinco adaptaciones reglamentarias resultado de combinar tres tipos de balón -números 5, 6 y 7- y dos alturas de la canasta -a 3,05 m. y a 2,80 m. del suelo-. Mediante análisis estadístico y la detección de patrones temporales se han concretado aspectos técnicos erróneos en determinadas modalidades sometidas a estudio, que nos permiten elevar propuestas intermedias de adaptación del baloncesto al niño de 11-12 años.

\section{ABSTRACT}

This study aims to raise an intermediate proposal for basketball in the children's category that will make the transition from mini-basketball to adult basketball progressive. To this end, we have studied the impacts on the free throw shooting technical pattern of five regulatory adaptations resulting from the combination of three types of ball -numbers 5, 6 and 7- and two basket heights -at $3.05 \mathrm{~m}$. and at $2.80 \mathrm{~m}$. from the ground-. Through statistical analysis and the detection of temporary patterns, erroneous technical aspects have been established in certain modalities under study, which enables us to raise intermediate proposals for adaptation of basketball for 1112 year olds. 


\section{INTRODUCCIÓN}

A partir del estudio del tiro libre -una conducta motriz por la que todos los jugadores, comúnmente, se van a enfrentar en un partido de baloncesto ${ }^{1}$ - pretendemos elevar una propuesta alternativa de juego de baloncesto que constituya un paso intermedio entre la categoría alevín y la cadete, ajustándose más a las posibilidades motrices del niño que a los requerimientos hegemónicos del baloncesto adulto.

La experiencia cotidiana de aquellos que se dedican al baloncesto base demuestra continuamente que el paso de la modalidad de minibasket practicada en la categoría alevín (1012 años) al baloncesto practicado en la categoría infantil (12-14 años), supone un salto excesivamente brusco para el cual el niño en ocasiones no se encuentra preparado ${ }^{2-4}$.
Y es que el niño pasa de jugar con balón no 5 y una altura de canasta de $2,60 \mathrm{~m}$. a utilizar el mismo balón -el no 7- y altura de canasta -3,05 m.- con la que se compite en el baloncesto de élite.

De esta forma, en el presente trabajo se someten a estudio los efectos en el patrón técnico de lanzamiento de tiro libre -en relación al tiro libre de la modalidad alevín y al modelo ideal de tiro libre- de posibles adaptaciones reglamentarias (véase tabla 1). Estas adaptaciones, resultan como consecuencia de combinar tres tipos de balón -alevín (no 5), adulto féminas ( $n^{\circ} 6$ ) y adulto masculino ( $n^{\circ} 7$ ) - y dos alturas de la canasta -la correspondiente a la categoría infantil y al baloncesto adulto (a $3,05 \mathrm{~m}$. del suelo) y otra intermedia entre la modalidad alevín y la infantil (a 2,80 m. del suelo)-.

Tabla 1. Situaciones de tiro libre sometidas a estudio.

\begin{tabular}{|c|c|c|c|c|}
\hline & & $\begin{array}{l}\text { Altura de la } \\
\text { canasta }\end{array}$ & $\begin{array}{c}\text { Tamaño de } \\
\text { balón }\end{array}$ & $\begin{array}{c}\text { Distancia de tiro } \\
\text { libre }\end{array}$ \\
\hline \multirow{7}{*}{ 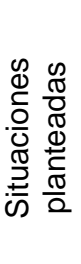 } & Alevín & $2,60 \mathrm{~m}$ & № 5 & $4 \mathrm{~m}$. del tablero \\
\hline & Infantil & $3,05 \mathrm{~m}$ & № 7 & $4,60 \mathrm{~m}$. del tablero \\
\hline & CIBA & $3,05 \mathrm{~m}$ & № 5 & $4,60 \mathrm{~m}$. del tablero \\
\hline & CIBP & $3,05 \mathrm{~m}$ & № 6 & $4,60 \mathrm{~m}$. del tablero \\
\hline & CPBA & $2,80 \mathrm{~m}$ & № 5 & $4,60 \mathrm{~m}$. del tablero \\
\hline & CPBI & $2,80 \mathrm{~m}$ & № 7 & $4,60 \mathrm{~m}$. del tablero \\
\hline & CPBP & $2,80 \mathrm{~m}$ & № 6 & $4,60 \mathrm{~m}$. del tablero \\
\hline
\end{tabular}

Es relevante recordar que las modificaciones reglamentarias que en el presente trabajo se someten a estudio pueden llevarse a la práctica sin necesidad de modificar ni las instalaciones, ni los equipamientos homologados. De hecho, las canastas de baloncesto que existen en la gran mayoría de los polideportivos son regulables en altura desde un mínimo de 2,60 $\mathrm{m}$. hasta un máximo de $3,05 \mathrm{~m}$. $\mathrm{Y}$, como ya hemos referido, el balón no 6 es el correspondiente a la competición femenina, a partir de la categoría infantil.

\section{Método}

La presente investigación sigue una metodología cuasi-experimental y observacional, las cuales tal como está planteado el estudio, son complementarias. Nuestro estudio se fundamenta en la metodología cuasi-experimental debido a que realizamos una manipulación de variables -la altura de la canasta y el tamaño y peso del balón-, y al hecho de que la elección de la muestra no es aleatoria. Pero el hecho de que, en el presente estudio, la metodología observacional predomine sobre la cuasiexperimental, nos ha llevado a seguir un diseño observacional. En concreto, el diseño observacional que vamos a utilizar es: puntual de seguimiento intrasesional, nomotético y multidimensional.

\section{Participantes}

Los participantes en el presente trabajo de investigación son los jugadores integrantes de la Selección Riojana Alevín, 2005. Aunque, de acuerdo con la normativa oficial de los Campeonatos de España de Baloncesto Alevín, la selección objeto de estudio posee doce integrantes, nueve han sido los jugadores participantes en la investigación. Al respecto, una de las bajas se ha debido a la ausencia por lesión de uno de los jugadores, en la sesión concreta de recogida de los datos. Las otras dos bajas de la muestra, quedan justificadas por el hecho de que los jugadores desechados no cumplían todos los criterios prefijados de constancia inter-sesional ya que, en concreto, habían participado con anterioridad en competiciones oficiales en categoría infantil hecho normativamente válido-.

\section{Instrumento de Observación}

El instrumento de observación ha sido elaborado ad hoc, a partir de una amplia revisión teórica sobre la técnica de ejecución del tiro libre. Es una combinación de formato de

Rev. Ib. CL. Act. Fis. Dep. 
campo y sistema de categorías ${ }^{5}$, ya que todos los criterios vertebradores han dado lugar a un sistema de categorías, excepto el número ocho: "fotograma clave".
En la tabla 2 se expone el desarrollo esquemático de los criterios constitutivos del instrumento de observación.

Tabla 2. Descripción abreviada de los criterios del instrumento de observación.

\begin{tabular}{|c|c|c|c|c|}
\hline № & Criterio & \multicolumn{3}{|c|}{$\begin{array}{c}\text { Categorías } \\
\end{array}$} \\
\hline 1 & Jugador & \multicolumn{3}{|c|}{ Integrantes de la selección riojana alevín } \\
\hline 2 & Distancia de tiro & A 4 m. del tablero $(\mathrm{A})$ & \multicolumn{2}{|l|}{ A 4,60 m. del tablero (I) } \\
\hline 3 & $\begin{array}{l}\text { Altura de la } \\
\text { canasta }\end{array}$ & $2,60 \mathrm{~m} .(\mathrm{D})$ & \multicolumn{2}{|l|}{3,05 m. (M) } \\
\hline 4 & $\begin{array}{l}\text { Características del } \\
\text { balón }\end{array}$ & № 5 (BA) & № 6 (BP) & № 7 (BG) \\
\hline 5 & Tiro con salto & Tiro con salto $(\mathrm{Y})$ & \multicolumn{2}{|l|}{ Tiro sin salto $(\mathrm{N})$} \\
\hline \multirow[t]{2}{*}{6} & \multirow[t]{2}{*}{ Tipo de salto } & Sin salto (SN) & Adelante (SD) & \multirow[t]{2}{*}{ Vertical (SV) } \\
\hline & & Lateral (SL) & Atrás (ST) & \\
\hline \multirow[t]{2}{*}{7} & \multirow[t]{2}{*}{ Fotograma clave } & Fotograma no clave (FN) & Fotograma preparación (FP) & \multirow{2}{*}{$\begin{array}{l}\text { Fotograma subida del balón } \\
\text { (FS) }\end{array}$} \\
\hline & & $\begin{array}{l}\text { Fotograma antes del despegue } \\
\text { (FA) }\end{array}$ & Fotograma finalización (FF) & \\
\hline \multirow[t]{2}{*}{8.1} & \multirow[t]{2}{*}{$\begin{array}{l}\text { Alineación de los } \\
\text { pies }\end{array}$} & En el aire (AIRE) & Alineados (APPI) & $\begin{array}{l}\text { Pie correspondiente al brazo } \\
\text { ejecutor adelantado menos } \\
\text { de un pie (EAMN) }\end{array}$ \\
\hline & & $\begin{array}{l}\text { Pie correspondiente al brazo } \\
\text { ejecutor adelantado un pie o } \\
\text { más de un pie (EAMS) }\end{array}$ & $\begin{array}{l}\text { Pie correspondiente al brazo } \\
\text { de apoyo retrasado menos } \\
\text { de un pie (ERMN) }\end{array}$ & $\begin{array}{l}\text { Pie correspondiente al brazo } \\
\text { de apoyo retrasado un pie o } \\
\text { más de un pie (ERMS) }\end{array}$ \\
\hline \multirow[t]{2}{*}{8.2} & \multirow[t]{2}{*}{$\begin{array}{l}\text { Separación de } \\
\text { los pies }\end{array}$} & En el aire (SAI) & $\begin{array}{l}\text { Mayor que la anchura de los } \\
\text { hombros (SMY) }\end{array}$ & \multirow[t]{2}{*}{$\begin{array}{l}\text { Igual a la anchura de los } \\
\text { hombros }(\mathrm{SIH})\end{array}$} \\
\hline & & $\begin{array}{l}\text { Menor que la anchura de los } \\
\text { hombros (SMN) }\end{array}$ & Pies juntos (NSP) & \\
\hline \multirow[t]{4}{*}{8.3} & \multirow{4}{*}{$\begin{array}{l}\text { Orientación de } \\
\text { los pies }\end{array}$} & En el aire (AAII) & Hacia la canasta (PPCC) & \multirow{2}{*}{$\begin{array}{l}\text { Se "miran" entre sí (PPII) } \\
\text { Pie-brazo ejecutor hacia } \\
\text { canasta y pie-brazo de } \\
\text { apoyo hacia interior (ECAI) }\end{array}$} \\
\hline & & $\begin{array}{l}\text { Pies orientados hacia el exterior } \\
\text { (PPEE) }\end{array}$ & $\begin{array}{l}\text { Pie correspondiente al brazo } \\
\text { ejecutor hacia canasta y pie } \\
\text { correspondiente al brazo de } \\
\text { apoyo hacia el exterior } \\
(\text { ECAE) }\end{array}$ & \\
\hline & & $\begin{array}{l}\text { Pie-brazo ejecutor hacia interior } \\
\text { y pie-brazo de apoyo hacia } \\
\text { canasta (EIAC) }\end{array}$ & $\begin{array}{l}\text { Pie-brazo ejecutor hacia } \\
\text { interior y pie-brazo de apoyo } \\
\text { hacia exterior (EIAE) }\end{array}$ & $\begin{array}{l}\text { Pie-brazo } \text { ejecutor hacia } \\
\text { exterior y pie-brazo de } \\
\text { apoyo hacia canasta } \\
\text { (EEAC) }\end{array}$ \\
\hline & & \multicolumn{3}{|c|}{ Pie-brazo ejecutor hacia exterior y pie-brazo de apoyo hacia el interior (EEAI) } \\
\hline 9.1 & $\begin{array}{l}\text { Alineación del } \\
\text { balón }\end{array}$ & Delante del cuerpo (DL) & Detrás del cuerpo (DT) & En el cuerpo (EN) \\
\hline \multirow[t]{2}{*}{9.2} & \multirow[t]{2}{*}{ Altura del balón } & Entre tobillo y rodilla $(\mathrm{PR})$ & Entre rodilla y cadera $(\mathrm{RD})$ & Entre cadera y hombro $(\mathrm{CH})$ \\
\hline & & $\begin{array}{l}\text { Entre hombro y vértex de la } \\
\text { cabeza }(\mathrm{HC})\end{array}$ & \multicolumn{2}{|c|}{ Por encima de la cabeza (EC) } \\
\hline \multirow[t]{2}{*}{9.3} & \multirow{2}{*}{$\begin{array}{l}\text { Posición del } \\
\text { balón }\end{array}$} & Lado del brazo ejecutor (BLE) & Mitad ejecutora (BME) & Mitad de apoyo (BMA) \\
\hline & & Lado de apoyo (BLA) & & \\
\hline \multirow[t]{3}{*}{10} & \multirow[t]{3}{*}{$\begin{array}{l}\text { Rotación de los } \\
\text { hombros }\end{array}$} & $\begin{array}{l}\text { Grados positivos de uno a } \\
\text { quince grados (CQP) }\end{array}$ & $\begin{array}{l}\text { Grados positivos } \text { de } \\
\text { dieciséis a treinta grados } \\
\text { (QTP) }\end{array}$ & $\begin{array}{l}\text { Grados positivos de treinta y } \\
\text { uno a cuarenta y cinco } \\
\text { grados (TCP) }\end{array}$ \\
\hline & & $\begin{array}{l}\text { Grados positivos de cuarenta y } \\
\text { seis a sesenta grados (CSP) }\end{array}$ & $\begin{array}{l}\text { Grados positivos de sesenta } \\
\text { y uno a setenta y cinco } \\
\text { grados (SSP) }\end{array}$ & $\begin{array}{l}\text { Grados positivos de setenta } \\
\text { y seis a noventa grados } \\
\text { (SNP) }\end{array}$ \\
\hline & & \multicolumn{3}{|c|}{ Grados negativos idem:\{(CQN), (QTN), (TCN), (CSN), (SSN), (SNN)\} } \\
\hline 11 & $\begin{array}{c}\text { Orientación } \\
\text { brazo ejecutor }\end{array}$ & A canasta $(\mathrm{BC})$ & Hacia el interior (BI) & Hacia el exterior (BE) \\
\hline 12. 1 & $\begin{array}{c}\text { Orientación de la } \\
\text { muñeca }\end{array}$ & Hacia canasta (MCA) & Hacia lado apoyo (MLA) & Hacia lado ejecutor (MLE) \\
\hline 12.2 & $\begin{array}{l}\text { Flexión de la } \\
\text { muñeca }\end{array}$ & Flexión completa (MFY) & \multicolumn{2}{|l|}{ Flexión incompleta (MFN) } \\
\hline 13 & $\begin{array}{l}\text { Extensión del } \\
\text { codo }\end{array}$ & Codo flexionado (CF) & \multicolumn{2}{|l|}{ Codo extendido (CE) } \\
\hline 14 & Eficacia & Entra $(\mathrm{T})$ & \multicolumn{2}{|l|}{ Falla (F) } \\
\hline
\end{tabular}

\section{Procedimiento}

La filmación de los tiros constitutivos del estudio se realizó en el seno de un entrenamiento de la Selección Alevín, tras realizar un calentamiento ordinario que fue filmado para contrarrestar el sesgo de reactividad ${ }^{6}$. Todos los tiros se grabaron con tres cámaras: frontal, lateral y cenital -véase figura 1-. Se garantizó la equivalencia métrica de las imágenes captadas por las cámaras frontal y lateral.

Rev. Ib. Cᄃ. Act. Fis. Dep. 
Estudio de propuestas intermedias de baloncesto en categaría infantil Garzón et al.

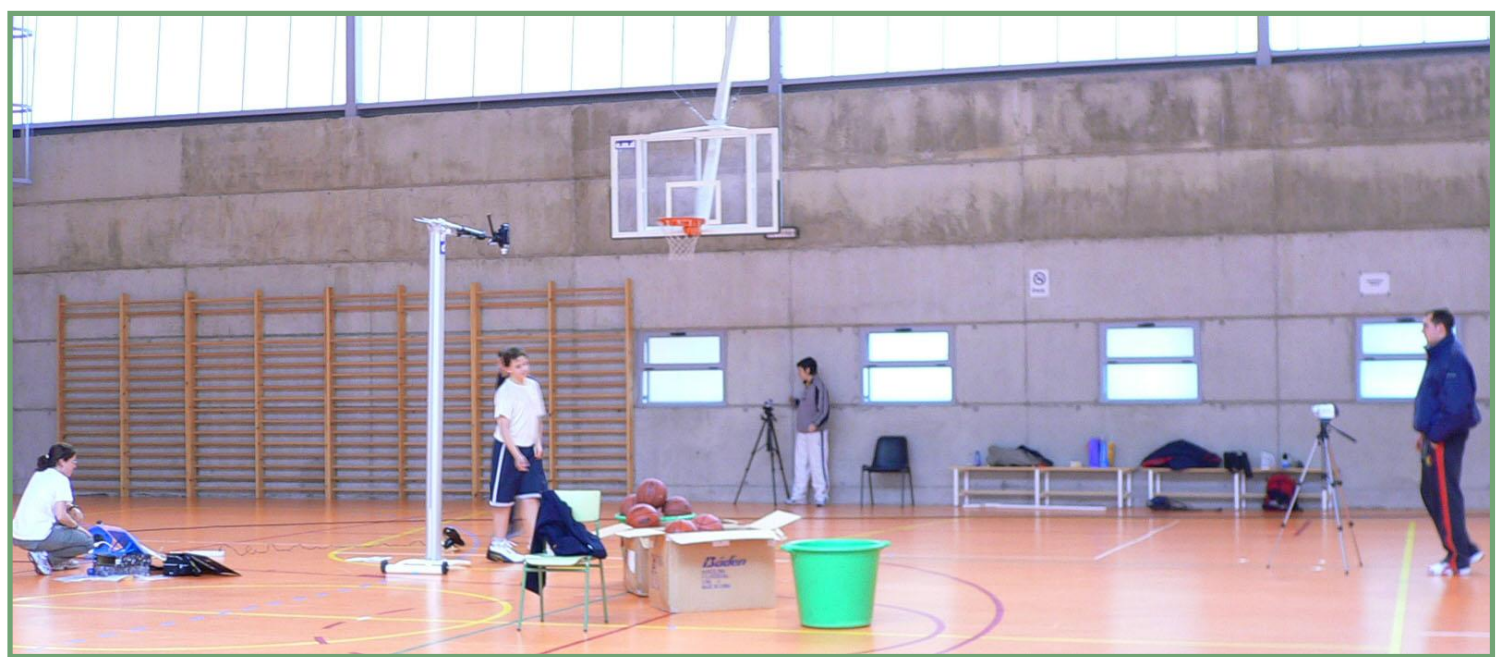

Figura 1. Posición de las tres cámaras con las que se filmaron los tiros libres.

Todos los jugadores participantes en el estudio realizaron diez lanzamientos en cada una de las situaciones descritas en la tabla 1 . Se filmaron un total de seiscientos treinta tiros, setenta tiros por cada uno de los nueve participantes estudiados.

Con la finalidad de evitar la influencia de las diferencias de nivel y experiencia de los jugadores, así como del posible proceso de aprendizaje derivado de la ejecución de los tiros, se llevó a cabo un balanceo de grupos equivalentes incompleto ${ }^{7}$.

\section{Registro y codificación}

El tipo de datos que manejamos, a partir de la terminología acuñada por Bakeman $(1978)^{8}$, son concurrentes tiempo-base (Tipo IV).

El registro de los lanzamientos se ha realizado mediante el programa ThemeCoder -véase figura 2-, teniendo en cuenta las consideraciones expuestas en Garzón, Lapresa, Anguera y Arana $(2011)^{9}$, utilizando un registro sistematizado con códigos mixtos: literales y numéricos.

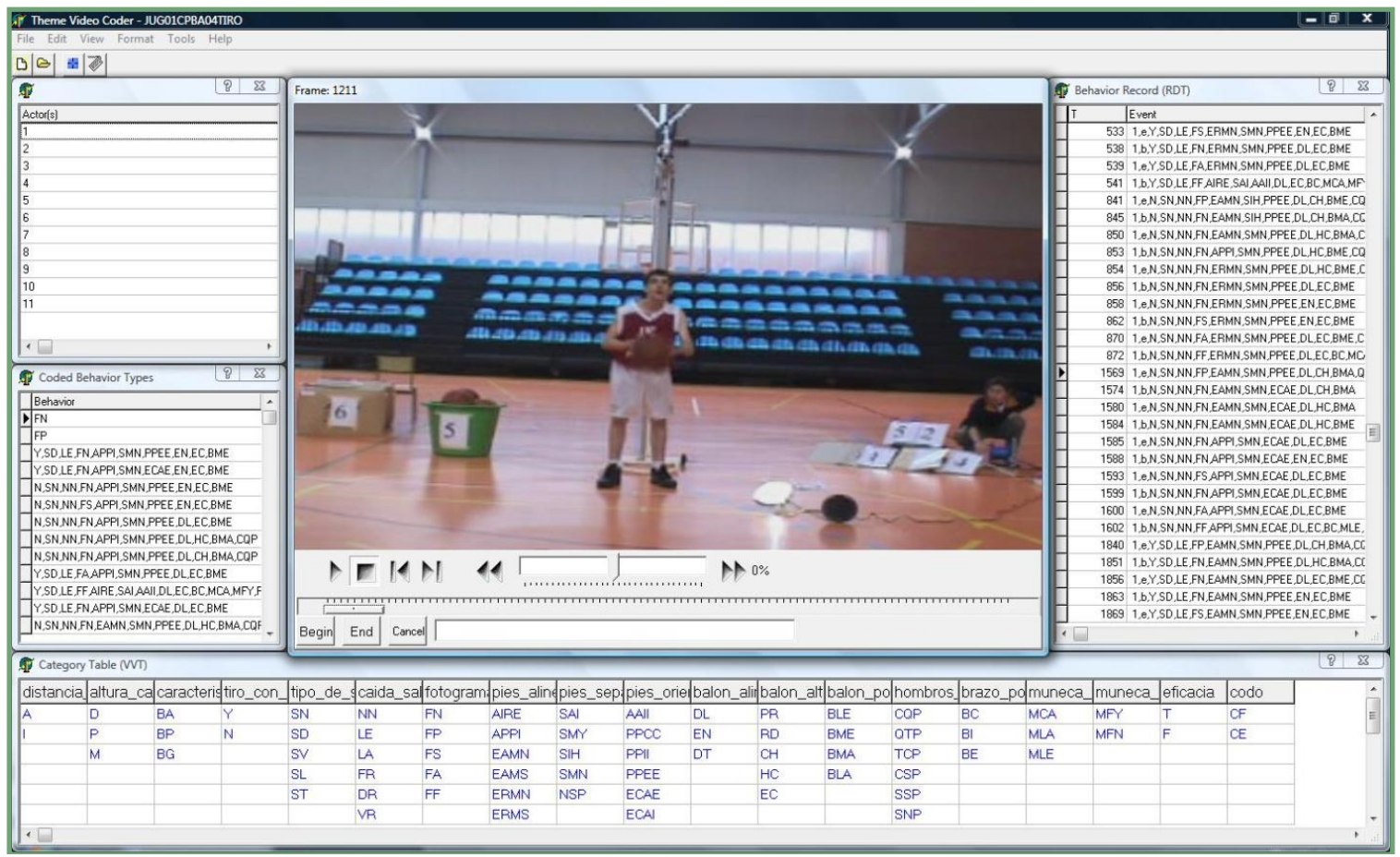

Figura 2. Captura del proceso de registro mediante ThemeCoder

Rev. Ib. Cᄃ. Act. Fis. Dep. 


\section{De la calidad del dato}

\section{Concordancia entre las observaciones}

Se ha llevado a cabo una concordancia intraobservador, mediante el cálculo del coeficiente Kappa de Cohen, a través del programa informático SDIS-GSEQ, versión 5.0.
De la totalidad de los tiros libres efectuados, 1/3 han sido analizados en dos ocasiones: los efectuados por los tres primeros jugadores registrados. En la tabla 3, se expone el Kappa de Cohen por jugador.

Tabla 3. Valor del kappa de los tres participantes analizados.

\begin{tabular}{lccc}
\cline { 2 - 4 } & Jugador $\mathbf{1}$ & Jugador $\mathbf{2}$ & Jugador $\mathbf{3}$ \\
\hline Kappa de Cohen & 0,88 & 0,76 & 0,77 \\
\hline
\end{tabular}

Estos resultados traducen una elevada estabilidad entre las observaciones, ya que el valor del Kappa de Cohen es, para los tres jugadores, superior a 0,75.

\section{Generalizabilidad de los resultados}

Con el fin de profundizar sobre la calidad del dato, en el seno de la Teoría de la Generalizabilidad $(T G)^{10}$, se ha planteado un diseño de 4 facetas (balón, altura de la canasta, macrocategoría y jugadores: $B A M / J)$, realizándose la estimación de los componentes de varianza de forma aleatoria infinita para todos los criterios.

El análisis de los coeficientes de generalizabilidad en esta estructura de diseño, determina que se consigue una fiabilidad de precisión de generalización de 0,987. El elevado coeficiente de generalización obtenido nos sirve, además, para justificar el muestreo por conveniencia adoptado para el presente trabajo de investigación ${ }^{11}$.

\section{Análisis de los datos}

Se han realizado dos tipos de análisis: uno estadístico descriptivo -mediante el programa SPSS, versión 15.0.-, y otro en busca de patrones temporales -mediante el programa Theme, versión 5.0.-

Para la detección de patrones temporales se ha partido del análisis de cada jugador por modalidad de estudio. Como parámetro de búsqueda se ha seleccionado un nivel de significación de ,005. El patrón temporal detectado es aceptado si Theme encuentra, entre todas las relaciones generadas aleatoriamente, $n$ relaciones -con $(\mathrm{n} / 2000)<$ 0,005- como intervalo crítico con intervalos internos del mismo tamaño o más pequeños que los de la relación contrastada.

\section{RESULTADOS}

Por su pertinencia, se exponen las características de un patrón correcto de lanzamiento de tiro libre, a partir de la revisión -a la luz de los criterios del instrumento de observación- de la propuesta ideal de gesto técnico ${ }^{12-24}$. En concreto, el patrón ideal de tiro libre, se corresponde con un lanzamiento sin salto. Con "el pie correspondiente al brazo ejecutor adelantado menos de un pie" (EAMN) o con ambos "pies alineados" (APPI). Con una separación de los pies "igual a la anchura de los hombros" (SIH) o "menor a la anchura de los hombros" (SMN). Con el "pie correspondiente al brazo ejecutor hacia canasta y pie correspondiente al brazo de apoyo hacia el exterior" (ECAE) o con ambos pies orientados "hacia la canasta" (PPCC). Con el balón colocado a lo largo de la ejecución del tiro en "mitad ejecutora" (BME) y con los hombros en un rango de rotación positiva de uno a quince grados (CQP). Por último, en el fotograma de finalización, el brazo ejecutor se orienta "a canasta" (BC), con el "codo extendido" (CE), y una "flexión completa" de muñeca (MFY). A modo de ejemplo de ejecución técnica correcta se expone el dendograma de un patrón temporal correspondiente al jugador 2 , en la modalidad alevín. (Véase figura 3).

Rev. lb. Сᄃ. Act. Fis. Dep. 


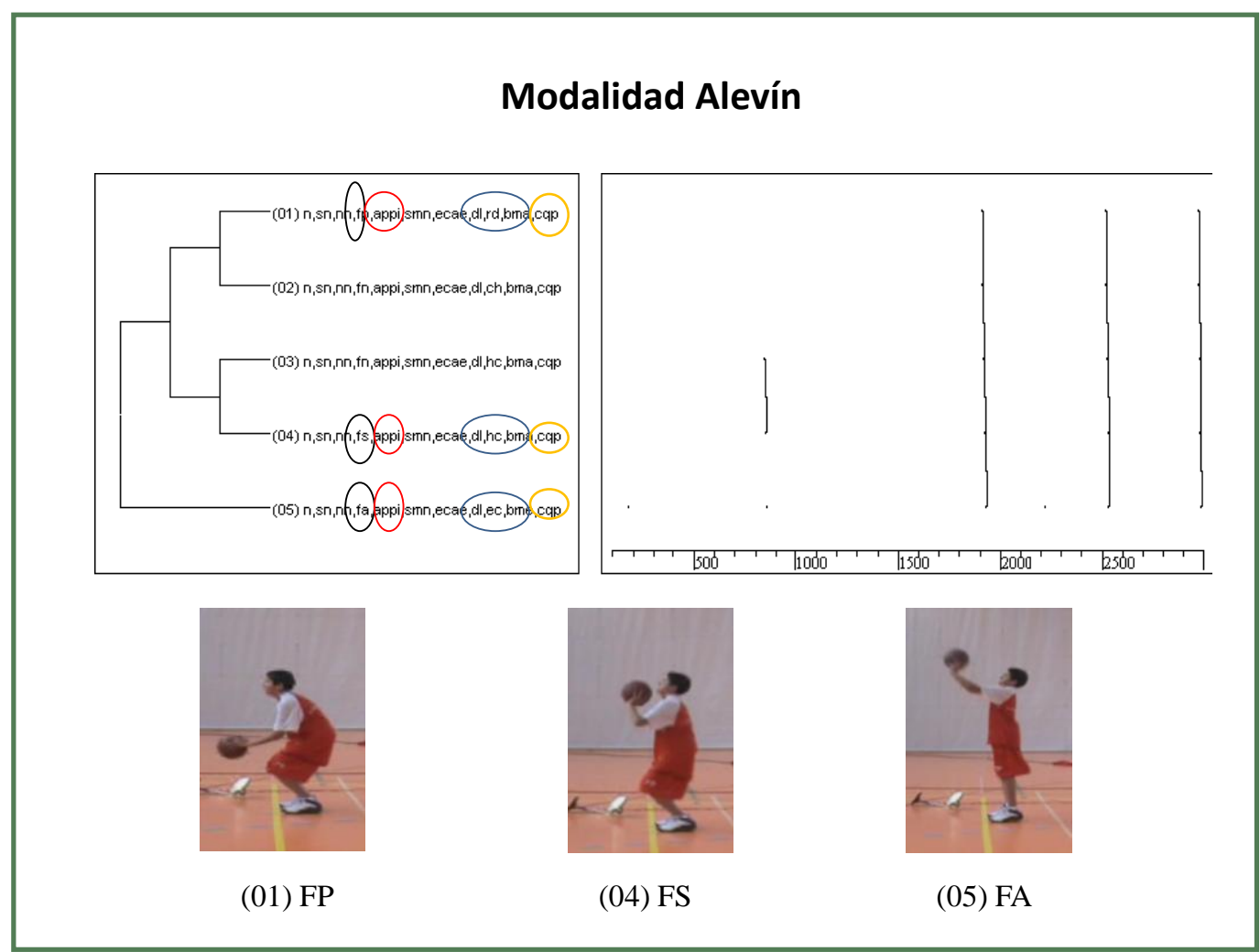

Figura 3. Patrón temporal correspondiente al jugador número 2 -modalidad alevín-. En color negro se resalta el fotograma del tiro del que se expone fotografía; en color rojo, la alineación de los pies; en color azul, las categorías relativas a la alineación, altura y posición del balón; y en color naranja, la rotación de los hombros.

\section{De la estadística descriptiva}

A continuación, se presentan resultados estadísticos relevantes relativos a los criterios del instrumento de observación que evidencian que, en la ejecución de los tiros libres correspondientes a las siete modalidades sometidas a estudio-, se producen errores técnicos concretos derivados de la desadaptación de la tarea a realizar.

La situación alevín es aquella en la que los tiros libres sin salto se presentan en mayor porcentaje (83\%). Le siguen las modalidades que combinan el balón de menor tamaño y peso -balón $n^{\circ}$ 5- con las alturas de canasta propuesta (CPBA) e infantil (CIBA), con un $57,8 \%$ y $54,4 \%$, respectivamente. El resto de modalidades muestran unos porcentajes muy similares de tiros con y sin salto. (Véase tabla 4).

Tabla 4. Tabla de contingencia de la relación entre modalidad y salto en el tiro libre.

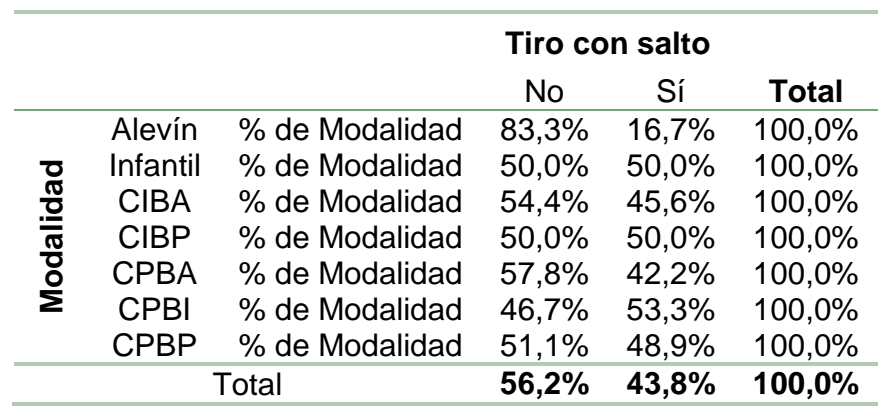

La modalidad alevín es aquella en la que un mayor porcentaje de tiros con salto $(58,3 \%)$ inician la fase aérea en el fotograma "finalización". Además, resulta relevante que la Rev. lb. CL. Act. Fis. Dep. modalidad alevín no anticipe ningún salto al fotograma "antes del despegue". En el polo contrario, la situación infantil es la que presenta un mayor porcentaje de saltos que inician con 
anterioridad al fotograma "antes del despegue" $11,4 \%$-. En las modalidades intermedias sometidas a estudio, la fase aérea del salto se inicia con mayor frecuencia en el fotograma "antes del despegue" -a excepción de la situación CIBA que acontece con posterioridad al fotograma "antes del despegue"-. (Véase tabla 5)

Tabla 5. Tabla de contingencia de la relación entre modalidad y fotograma en el que se inicia la fase aérea del salto en el tiro libre. Siendo: FN -"fotograma no clave"-; FA -fotograma "antes del despegue"-; y FF -fotograma "finalización"-.

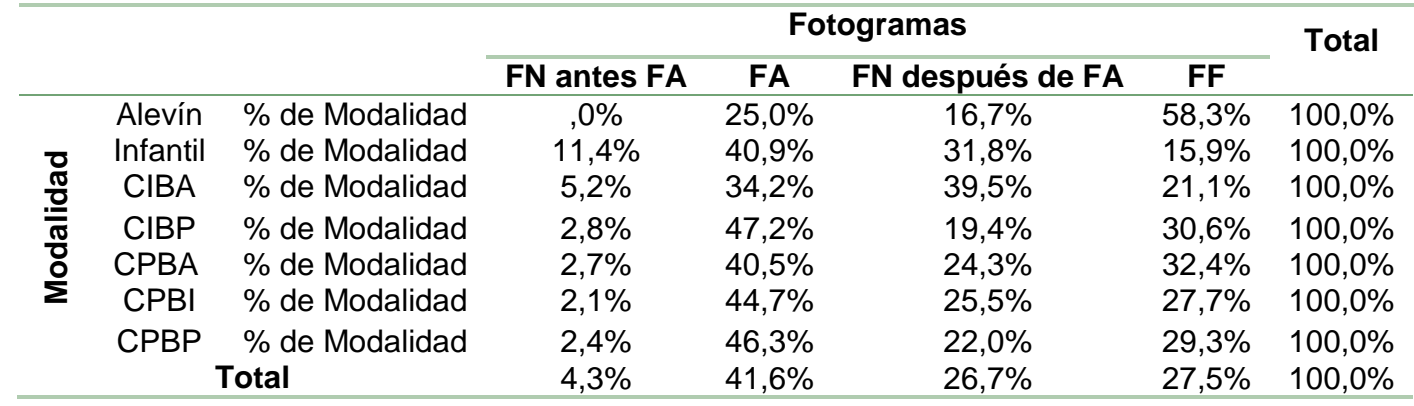

La modalidad alevín es la única de todas las contempladas en el presente trabajo de investigación en la que no se han registrado angulaciones en grados negativos. Por otro lado, la situación infantil es la que mayor porcentaje de rangos de rotación negativos registra $(9,4 \%)$. Además, en comparación con la modalidad alevín, en el resto de modalidades se detecta un aumento de la frecuencia de aparición de angulaciones de dieciséis a treinta grados positivos (QTP) y la introducción de rangos negativos de rotación -de cero a quince grados (CQN)-. (Véase tabla 6)

Tabla 6. Tabla de contingencia de la relación entre modalidad y rotación de los hombros en el tiro libre.

\begin{tabular}{|c|c|c|c|c|c|c|c|}
\hline & & & \multicolumn{4}{|c|}{ Rotación de los hombros } & \multirow{2}{*}{ Total } \\
\hline & & & CQP & QTP & TCP & CQN & \\
\hline \multirow{8}{*}{ 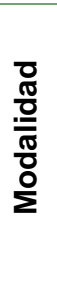 } & Alevín & $\%$ de Modalidad & $78,2 \%$ & $19,9 \%$ & $1,9 \%$ &, $0 \%$ & $100,0 \%$ \\
\hline & Infantil & $\%$ de Modalidad & $67,0 \%$ & $22,3 \%$ & $1,3 \%$ & $9,4 \%$ & $100,0 \%$ \\
\hline & CIBA & $\%$ de Modalidad & $74,5 \%$ & $23,0 \%$ &, $2 \%$ & $2,3 \%$ & $100,0 \%$ \\
\hline & CIBP & $\%$ de Modalidad & $71,6 \%$ & $25,0 \%$ & $1,4 \%$ & $2,0 \%$ & $100,0 \%$ \\
\hline & CPBA & $\%$ de Modalidad & $73,7 \%$ & $23,5 \%$ & ,2\% & $2,6 \%$ & $100,0 \%$ \\
\hline & CPBI & $\%$ de Modalidad & $72,3 \%$ & $22,9 \%$ & ,2\% & $4,6 \%$ & $100,0 \%$ \\
\hline & CPBP & $\%$ de Modalidad & $75,4 \%$ & $19,5 \%$ &, $0 \%$ & $5,0 \%$ & $100,0 \%$ \\
\hline & \multicolumn{2}{|c|}{ Total } & $73,2 \%$ & $22,3 \%$ & ,7\% & $3,8 \%$ & $100,0 \%$ \\
\hline
\end{tabular}

La modalidad alevín es la que presenta menor porcentaje $(82,2 \%)$ de tiros en los que, en el fotograma "finalización", se extiende por completo el codo. En el polo contrario (92,2\%), nos encontramos con la modalidad infantil véase tabla 7-.

Tabla 7. Tabla de contingencia de la relación entre modalidad y flexión del codo en el tiro libre.

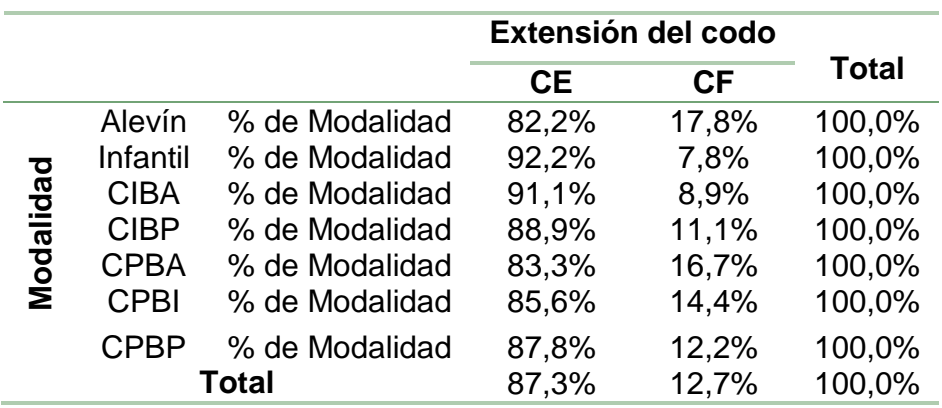

Rev. Ib. CL. Act. Fis. Dep. 
Además, en todas las situaciones de estudio excepto en la situación alevín (84,4\%)-, el porcentaje de tiros en los que se flexiona la muñeca sobrepasa el $90 \%$. (Véase tabla 8 )

Tabla 8. Tabla de contingencia de la relación entre modalidad y flexión de la muñeca en el tiro libre.

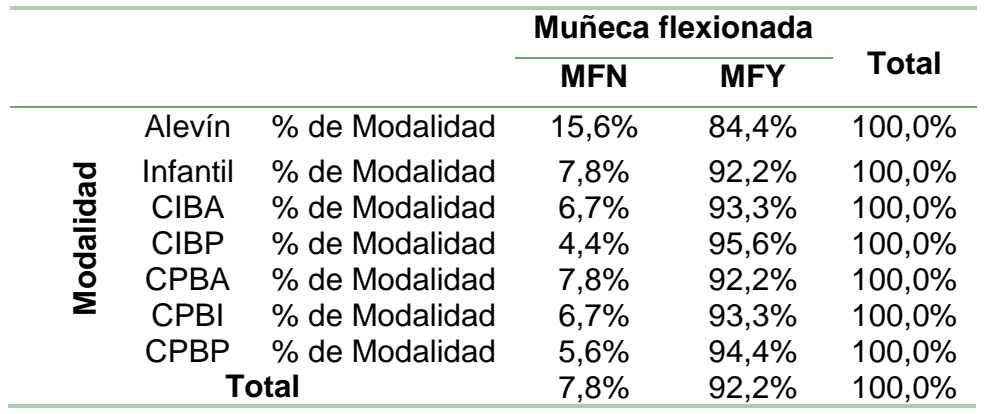

\section{De los patrones temporales}

A continuación se abordan, a la luz del análisis estadístico realizado, aspectos técnicos relevantes relacionados con los principales errores detectados en los patrones temporales correspondientes a los lanzamientos de los jugadores en las modalidades sometidas a estudio.

a) Modalidad alevín

El jugador $n^{\circ} 8$, es el único lanzador que en sus patrones temporales únicamente refleja lanzamientos con salto. Por su parte, el jugador no 6 presenta patrones temporales con y sin salto. La fase aérea del salto se inicia en el fotograma "finalización" -jugador $\mathrm{n}$ ㅇ 8- o con posterioridad al mismo -jugador $\mathrm{n}$ - 6-, lo cual nos indica la brevedad y menor relevancia del salto. En cuanto a la altura del balón en el fotograma "subida del balón" la altura más baja detectada es "entre los hombros y la cabeza" excepto el jugador 10 que sitúa el balón "entre la cadera y los hombros" en todas las modalidades-.

En cuanto a la rotación de los hombros se detecta un rango que excede la categoría de cero a quince grados positivos, en los patrones temporales correspondientes a los jugadores $\mathrm{n}^{\circ}$ 7,8 y 10.

Por último, en la finalización del tiro, el único error detectado es la ausencia de flexión completa de la muñeca en patrones temporales correspondientes a los jugadores nำ 6, 8 y 11 .

b) Modalidad infantil

Los patrones correspondientes a los tiros libres infantiles incluyen tiros con salto en los jugadores no 1, 6, 8, y 9; además, en el jugador $\mathrm{n}^{-} 11$, se detectan patrones temporales tanto con salto como sin salto. De los jugadores que presentan patrones temporales con salto, la fase de vuelo se produce: en el jugador $n \div 6$ con anterioridad al fotograma "antes del despegue"; en los jugadores $n^{\circ} 8$ y 11 en el fotograma "antes del despegue"; y, en el jugador $n^{\circ} 1$, con posterioridad a este fotograma. En cuanto a la altura del balón en el fotograma "subida del balón", tres jugadores -jugadores ํㅜ 2, 9 y 10 colocan el balón "entre la cadera y los hombros". En lo relativo a la rotación de los hombros, se detectan angulaciones positivas de dieciséis a treinta grados en los patrones temporales de los jugadores $\mathrm{n}$ ㅇ 3, 7, 8, 10 y 11. Además, los jugadores $\mathrm{n}^{\circ} 10$ y 11 , presentan patrones temporales con rotaciones negativas, de cero a quince grados.

Por último, en el fotograma "finalización", el jugador $n^{0} 11$ presenta patrones temporales que señalan la ausencia de flexión completa de muñeca; mientras que en el no 6 se detecta el error que constituye el no extender el codo completamente.

\section{c) Modalidad CIBA}

En lo relativo a la inclusión de salto en el tiro libre, tres jugadores - $n^{\circ} 6,8$ y 9-presentan patrones temporales con salto; y el jugador $n \circ 1$ tanto con salto como sin él. La fase aérea del salto se detecta en los jugadores $n^{\circ} 6$ y 8 en el fotograma "antes del despegue" y en el jugador no 9 después de dicho fotograma. La altura del balón en el fotograma "subida del balón" se produce "entre la cadera y los hombros" en los patrones temporales de los jugadores $n=2,10, y$ 11.

En relación a la rotación de los hombros, decir que cuatro jugadores $-n^{\circ}=3,7,8,10$-, presentan patrones temporales con un rango de rotación positiva excesivo -de dieciséis a treinta grados-.

\section{d) Modalidad CIBP}

En lo relativo a la introducción de salto en el tiro libre, cuatro jugadores $-n-1,6,8$ y 9 presentan patrones temporales con salto; mientras que el jugador no 11 presenta patrones temporales que reflejan tiros con y sin salto. En lo relativo a la fase aérea del salto decir que, en los cuatro jugadores - $n^{\circ}=6,8,9$ y 11 - en los que se detecta la fase de vuelo, se inicia en el fotograma "antes del despegue" o con posterioridad a este fotograma. La altura del balón en el fotograma "subida del balón" se

$$
\text { Rev. lb. CL. Act. Fis. Dep. }
$$


produce "entre la cadera y los hombros" en los patrones temporales correspondientes a tres jugadores - $n=2,9$, y 10-

En cuanto a la rotación de los hombros, seis jugadores $-n^{\circ}=3,6,7,8,10,11$ - presentan patrones temporales con un rango de rotación positiva excesivo -de dieciséis a treinta grados-. En el fotograma "finalización", únicamente se detecta el error técnico de falta de flexión completa de la muñeca en el jugador $\mathrm{n} \times 11$.

\section{e) Modalidad CPBA}

En lo relativo a la inclusión de salto en el lanzamiento: tres jugadores presentan patrones temporales con salto -no 6,8 y $9-;$ y otros tres participantes $-n^{\circ} 1,7$, y 11 -, patrones que incluyen tiros con $y$ sin salto. En cuatro jugadores $-n^{\circ} 6,7,8$ y 11 - en los que se detecta la fase aérea del salto, ésta se inicia en el fotograma "antes del despegue" 0 con posterioridad a este fotograma; y en el jugador 9 en el fotograma "finalización". La altura del balón en el fotograma "subida del balón" se produce entre la cadera y los hombros en los patrones de los jugadores no 2 y 10 .

En relación a la rotación de los hombros, el rango de rotación positiva de dieciséis a treinta grados se refleja en los patrones relativos a los jugadores $n^{\circ} 3,6,7,8$, y 10 . Además, en el jugador $n^{\circ} 2$, se han detectado patrones con rangos de rotación positivos y negativos. La rotación negativa detectada es de cero a quince grados.

En el fotograma "finalización", únicamente se detecta el error técnico de falta de extensión completa del codo en el jugador $n^{\circ} 1$.

\section{f) Modalidad CPBI}

En lo relativo a la inclusión de salto en el lanzamiento cuatro jugadores - $n^{\circ}=1,6,8$ y 9presentan patrones temporales con salto; mientras que el jugador no 11 presenta patrones temporales que reflejan tiros con y sin salto. La fase aérea del salto se detecta en tres jugadores -no 6, 8 y 11- en el fotograma "antes del despegue" y en los participantes $\mathrm{n}$ ㅇ 1 y 9 después de este fotograma -el jugador $n-1$ en el fotograma "finalización"-. La altura del balón en el fotograma "subida del balón" se produce "entre la cadera y los hombros" en los patrones de los jugadores $\mathrm{n}^{\circ} 2$ y 10 .

En relación a la rotación de los hombros el rango de dieciséis a treinta grados positivos se refleja en los patrones temporales relativos a los jugadores $n^{\circ}$ 6, 7 y 10 . Además, en el jugador $n^{\circ}$ 2 , se han detectado patrones temporales que incluyen rangos de rotación positiva y negativa. La rotación negativa detectada es de cero a quince grados.

En el fotograma "finalización", únicamente se detecta el error técnico de falta de extensión completa del codo en el jugador $n=1$.

\section{g) Modalidad CPBP}

En lo relativo a la inclusión de salto en el lanzamiento cuatro jugadores -no $1,6,8$ y 9 presentan patrones con salto; y el jugador no 11 obtiene patrones que incluyen tiros con y sin salto. En lo relativo a la fase aérea del salto decir que se detecta en los jugadores no 6,8 y 9 y que, en todos ellos, se inicia en el fotograma "antes del despegue" o con posterioridad a este fotograma. La altura del balón en el fotograma "subida del balón", se produce entre la cadera y los hombros en patrones temporales correspondientes al jugador $n^{0} 10$.

El rango de rotación positiva de dieciséis a treinta grados se refleja en patrones temporales de cuatro jugadores -no 6, 7, 8 y 10-. Además, en el jugador $n^{\circ} 11$ se detectan patrones temporales que incluyen rangos de rotación positiva y negativa -de cero a quince grados-.

En la tabla 9, se ofrece un resumen de los referidos aspectos relevantes que en cada modalidad se relacionan con la presencia de errores técnicos.

Rev. Ib. CL. Act. Fis. Dep. 
Tabla 9. Resumen de aspectos relevantes en la detección de errores técnicos, a partir de la información aportada por los patrones temporales.

\begin{tabular}{|c|c|c|c|c|c|c|}
\hline & \multicolumn{3}{|c|}{ Salto } & \multirow[b]{2}{*}{$\begin{array}{c}\text { Fotograma } \\
\text { "subida del } \\
\text { balón" }\end{array}$} & \multirow[b]{2}{*}{$\begin{array}{c}\text { Rotación } \\
\text { máxima de } \\
\text { hombros }\end{array}$} & \multirow[b]{2}{*}{$\begin{array}{c}\text { Fotograma } \\
\text { "finalización" }\end{array}$} \\
\hline & Sí & No & $\begin{array}{c}\text { Fotograma } \\
\text { inicio fase } \\
\text { aérea }\end{array}$ & & & \\
\hline ALV & $\begin{array}{l}\text { Jug: } 6 \\
\text { y } 8\end{array}$ & $\begin{array}{l}\text { Jug: } 1 \\
2,3,6 \\
7,9,10 \\
\text { y } 11\end{array}$ & $\begin{array}{l}\text { Jug 8: } \\
\text { Finalización } \\
\text { (FF) } \\
\text { Jug 6: } \\
\text { Después FF }\end{array}$ & $\begin{array}{l}\text { Jug 10: Entre } \\
\text { cadera y } \\
\text { hombros }(\mathrm{CH})\end{array}$ & $\begin{array}{l}\text { Jug } 7,8 \text { y } 10: \text { de } \\
\text { dieciséis a } \\
\text { treinta grados } \\
\text { positivos (QTP) }\end{array}$ & $\begin{array}{l}\text { Jug } 6,8 \text { y 11: } \\
\text { Flexión incompleta } \\
\text { de muñeca (MFN) } \\
\text { Jug 6: Extensión } \\
\text { incompleta del codo } \\
\text { (CF) }\end{array}$ \\
\hline CIBA & $\begin{array}{l}\text { Jug: } 1 \\
6,8 \text { y } \\
9\end{array}$ & $\begin{array}{l}\text { Jug: } 1 \\
2,3,7 \\
10 \text { y } 11\end{array}$ & $\begin{array}{l}\text { Jug } 6 \text { y } 8: \\
\text { Antes del } \\
\text { despegue (FA) } \\
\text { Jug 9: después } \\
\text { FA }\end{array}$ & $\begin{array}{l}\text { Jug 2, } 10 \text { y } 11: \\
\mathrm{CH}\end{array}$ & $\begin{array}{l}\text { Jug 3, 7, } 8 \text { y 10: } \\
\text { QTP }\end{array}$ & \\
\hline CIBI & $\begin{array}{l}\text { Jug: } 1 \\
6,8,9 \\
\text { y } 11\end{array}$ & $\begin{array}{l}\text { Jug: } 2 \\
3,7,10 \\
\text { y } 11\end{array}$ & $\begin{array}{l}\text { Jug 6: antes } \\
\text { FA } \\
\text { Jug } 8 \text { y 11: FA } \\
\text { Jug 1: después } \\
\text { FA }\end{array}$ & $\begin{array}{l}\text { Jug 2, } 9 \text { y 10: } \\
\mathrm{CH}\end{array}$ & $\begin{array}{l}\text { Jug 3, 7, 8, } 10 \text { y } \\
\text { 11: QTP } \\
\text { Jug } 10 \text { y 11: de } \\
\text { uno a quince } \\
\text { grados negativos } \\
\text { (CQN) }\end{array}$ & Jug 11: MFN \\
\hline CIBP & $\begin{array}{l}\text { Jug: } 1 \\
6,8,9 \\
\text { y } 11\end{array}$ & $\begin{array}{l}\text { Jug: } 2 \\
3,7,10 \\
\text { y } 11\end{array}$ & $\begin{array}{l}\text { Jug 6, 8, } 9 \text { y } \\
11: \text { FA o } \\
\text { después FA }\end{array}$ & $\begin{array}{l}\text { Jug 2, } 9 \text { y 10: } \\
\mathrm{CH}\end{array}$ & $\begin{array}{l}\text { Jug 3, 6, 7, 8, } 10 \\
\text { y 11: QTP }\end{array}$ & Jug 11: MFN \\
\hline CPBA & $\begin{array}{l}\text { Jug: } 1 \\
6,7,8 \\
9 \text { y } 11\end{array}$ & $\begin{array}{l}\text { Jug: } 1 \\
2,3,7 \\
10 \text { y } 11\end{array}$ & $\begin{array}{l}\text { Jug } 6,7,8 \text { y } \\
\text { 11: FA o } \\
\text { después FA: } \\
\text { Jug 9: FF }\end{array}$ & Jug 2 y $10: \mathrm{CH}$. & $\begin{array}{l}\text { Jug 3, 6, 7, } 8 \text { y } \\
\text { 10: QTP } \\
\text { Jug 2: CQN }\end{array}$ & Jug 1: CF \\
\hline CPBI & $\begin{array}{l}\text { Jug: } 1 \\
6,8,9 \\
\text { y } 11\end{array}$ & $\begin{array}{l}\text { Jug: } 2 \\
3,7,10 \\
\text { y } 11\end{array}$ & $\begin{array}{l}\text { Jug } 6,8 \text { y 11: } \\
\text { FA } \\
\text { Jug } 1 \text { y 9: } \\
\text { después de FA } \\
\text { Jug 1: FF }\end{array}$ & Jug 2 y $10: \mathrm{CH}$ & $\begin{array}{l}\text { Jug 6, } 7 \text { y 10: } \\
\text { QTP } \\
\text { Jug 2: CQN }\end{array}$ & Jug 1: CF \\
\hline CPBP & $\begin{array}{l}\text { Jug: } 1 \\
6,8,9 \\
\text { y } 11\end{array}$ & $\begin{array}{l}\text { Jug: } 2 \\
3,7,10 \\
\text { y } 11\end{array}$ & $\begin{array}{l}\text { Jug } 6,8 \text { y 9: } \\
\text { FA o después } \\
\text { FA }\end{array}$ & Jug 10: CH & $\begin{array}{l}\text { Jug 6, 7, } 8 \text { y 10: } \\
\text { QTP } \\
\text { Jug 11: CQN }\end{array}$ & \\
\hline
\end{tabular}

\section{DISCUSIÓN}

La descripción técnica ideal del tiro libre no deja lugar a dudas de que debe ser un lanzamiento sin salto ${ }^{15,16,21,23,25}$. Y es que, la inclusión de salto en el tiro libre se encuentra directamente relacionada con los requerimientos de fuerza del lanzador para alcanzar con garantías la canasta. De esta forma, en la modalidad alevín es en la que en menor medida se ve reflejado este error tipo; mientras, en la categoría infantil y en el resto de modalidades, aumenta considerablemente la presencia del error que supone la categoría "salto" en el lanzamiento.

En lo relativo a la fase aérea del salto decir que, en los patrones temporales correspondientes a los dos jugadores que utilizan el salto en la modalidad alevín, se inicia en el fotograma "finalización" o con posterioridad al citado fotograma. Este hecho, refrendado por los resultados estadísticos, denota la brevedad y menor relevancia de la presencia del salto en el lanzamiento de tiro libre alevín. En el resto de modalidades, el jugador se ve obligado a anticipar la fase aérea del salto con lo que este error técnico toma mayor repercusión. En concreto, es en la modalidad infantil donde se ha detectado una mayor anticipación del salto. Uno de los indicios que evidencian la falta de fuerza de los jugadores jóvenes para dotar al balón del impulso necesario para alcanzar la canasta, es la disminución en la altura del balón en el comienzo de la ejecución del tiro 14-16, 18, 19, ${ }^{23}$. Este aspecto se pone de manifiesto en el fotograma "subida del balón". En todas las modalidades alternativas sometidas a estudio la altura del balón desde la que se saca el lanzamiento es inferior a la mantenida en los tiros libres alevines.

Rev. Ib. Cᄃ. Act. Fis. Dep. 
En cuanto a la rotación de los hombros, la descripción técnica ideal del tiro libre implica una ligera rotación fruto de la posición adelantada del hombro ejecutor ${ }^{15,16,19}$. En todas las modalidades alternativas estudiadas, se produce un aumento del rango de rotación respecto a los tiros libres efectuados en la modalidad alevín, basada principalmente en angulaciones positivas de dieciséis a treinta grados.

A diferencia de la modalidad alevín en la que sólo se detectan rotaciones positivas, en la modalidad infantil -con dos jugadores- y en las modalidadades CPBA, CPBI y CPBP -con unose han encontrado patrones temporales que incluyen rotaciones negativas, de cero a quince grados. Cabe destacar que rotar en grados negativos es un indicio más de desadaptación de la modalidad y constituyen un aporte erróneo de fuerza para alcanzar la canasta ${ }^{16}$.

En la finalización del tiro cobra especial relevancia la actuación del brazo ejecutor; tanto en lo relativo a la extensión del codo, como a la flexión completa de la muñeca. En la situación alevín, se detecta cierto margen de aplicación de fuerza para dotar al balón de la velocidad suficiente para que alcance la canasta en lo relativo a la extensión del codo y la ausencia de flexión completa de la muñeca. En el polo contrario, nos encontramos con la modalidad infantil; en la que los participantes dependen de una mayor y más rápida extensión del codo en la fase de finalización, para dotar al balón de la velocidad de salida necesaria para alcanzar con garantías la canasta.

\section{CONCLUSIONES}

Fruto del análisis estadístico descriptivo realizado -en relación a los criterios del instrumento de observación-, y de la ulterior interpretación de los patrones temporales detectados a la luz de estos resultados-guía, se han detectado errores manifiestos en la ejecución técnica del tiro libre en las diferentes modalidades sometidas a estudio.

La modalidad alevín se muestra como la modalidad en la que menos errores técnicos se detectan en relación al gesto técnico correcto. En el otro polo, la modalidad en la que el lanzamiento requiere trasladar un mayor impulso al balón como es la modalidad infantil mayor altura de canasta y mayor tamaño y peso del balón-, es aquella en la que más se agudizan determinados errores técnicos.

En concreto, las dificultades para alcanzar con garantías la canasta se ven reflejadas en errores técnicos encaminados a aumentar el impulso que trasladar al balón como son: la presencia de salto y la anticipación de su fase de vuelo; la disminución en la altura del balón en el comienzo de la ejecución del tiro; el aumento del rango de rotación positiva; así como la introducción de rangos negativos -para tomar un impulso previo a la rotación positiva-

Las modalidades intermedias, también se caracterizan por presentar este tipo de errores, aunque se constituyen como propuestas que suponen una evolución más progresiva de las premisas del juego y por lo tanto se erigen como alternativas más adaptadas a las posibilidades concretas del niño en la edad sometida a estudio.

\section{REFERENCIAS BIBLIOGRÁFICAS}

1. Hudson JL. Prediction of basketball skill using biomechanical variables. Research Quartely for Exercise and Sport. 1985; 56(2): 115-121.

2. Esper PA. Estudio sobre el tamaño de balón ideal en baloncesto para los jugadores de 13-14 años. Lecturas: Educación Física y Deportes. 1999; 4(15). Disponible en http://www.efdeportes.com

3. Garzón B. Adaptando el baloncesto al niño de 12-13 años: análisis observacional del lanzamiento de tiro libre. Tesis doctoral. Logroño: Universidad de La Rioja; 2010.

4. Garzón B, Lapresa D, Anguera MT, Arana J. Del minibasket al baloncesto: efectos de la actual configuración reglamentaria en el patrón técnico de lanzamiento de tiro libre. Revista de Psicología del Deporte. 2014; 23(1): 77-85.

5. Anguera MT, Magnusson MS, Jonsson GK. Instrumentos no estándar. Avances en medición. 2007; 5(1): 63-82.

6. Anguera MT. Manual de prácticas de observación. México: Trillas; 1983.

7. Arnau J. Diseños experimentales. Barcelona: Edicions Universitat de Barcelona; 2001.

8. Bakeman R. Untangling streams of behavior: Sequential analysis of observation data. In Sackett GP (Ed.) Observing Behavior, Vol. 2: Data collection and analysis methods. Baltimore: University of Park Press; 1978. p. 63-78.

9. Garzón B, Lapresa D, Anguera MT, Arana J. Análisis observacional del lanzamiento de tiro libre en jugadores de baloncesto base. Psicothema. 2011; 23(4): 851-857.

10. Cronbach LJ, Gleser GC, Nanda $H$, Rajaratnam N. The dependability of behavioral measurements: theory of generalizability for scores and profiles. New York: Wiley; 1972.

11. Anguera MT, Arnau J, Ato M, Martínez R, Pascual J, Vallejo G. Métodos de investigación en psicología. Madrid: Síntesis; 1995.

12. Peyró R, Sampedro J. Pedagogía del baloncesto. Valladolid: Miñón; 1979.

Rev. lb. Cᄃ. Act. Fis. Dep. 
13. Ripoll H, Bard C, Paillard J. Stabilization of head and eyes on target as a factor in successful basketball shotting. Human Movement Science. 1986; 5: 47-58.

14. Coloma M, Brizuela J. Iniciación al baloncesto. Zaragoza: Imagen y Deporte; 1997.

15. Alderete JL, Osma JJ. Baloncesto. Técnica individual de ataque. Madrid: Gymnos; 1998.

16. Torres A, Arjonilla N. Fundamentos individuales. Curso de entrenador de baloncesto de primer nivel. Madrid. FEB; 1998.

17. Krause J. Basketball skills \& drills. Champaign: Human Kinetics; 1999.

18. Faucher DG. Enseñar baloncesto a los jóvenes. Barcelona: Paidotribo; 2002.

19. Refoyo I. Técnica individual. En Escuela Nacional de Entrenadores (Eds.) Curso de iniciación al baloncesto. Madrid: Federación Española de Baloncesto; 2002. p. 11-27.

20. Wootten $M$. Coaching basketball successfully. Champaign: Human Kinetics; 2003.

21. Oliver J. Basketball fundamentals. A better way to learn the basics. Champaign: Human Kinetics; 2004.

22. American Sport Education Program. Coaching basketball technical and tactical skills. Champaign: Human Kinetics; 2007.

23. Wisel H. Shooting techniques. Champaign: Human Kinetics; 2009.

24. Montero A. Didáctica del baloncesto. Barcelona: Paidotribo; 2010.

25. Ibáñez SJ, García J, Feu S, Parejo I, Cañadas M. La eficacia del lanzamiento a canasta en la NBA: Análisis multifactorial. Cultura, Ciencia y Deporte. 2009; 5(10): 3947.

Referencias totales citadas: 25 .

Referencias citadas correspondientes a la Rev Ib CC Act Fis Dep: 0.

Rev. Ib. CL. Act. Fis. Dep. 